\title{
Seeking a better (residency) match
}

$\mathrm{M}$ atch day has come and gone, and most Canadian medical students have received good news. Across the country, final-year medical students recently underwent a six-month application process to gain admission to one of Canada's 17 postgraduate medical education programs for residency training. Choosing from 32 disciplines, about $95 \%$ of these students successfully matched into their first-choice specialty and about $60 \%$ matched into their first-choice program at their first-choice institution.

Despite this success, the match process itself is less than ideal, according to several trainee associations and some students applying for postgraduate training in Canada. Major concerns include limited opportunities for electives during medical school and a lack of transparency in the selection process. It's also timeconsuming to prepare for multiple interviews and expensive to travel to them.

To apply for residency, students make multiple applications to different universities through the Canadian Resident Matching Service (CaRMS) electronic interface and then attend interviews across the country. However, using a complex matching algorithm, whereby universities rank students in order of preference and vice-versa, students get only one job offer on match day. A minority of students (less than 5\%) do not match after round one and enter round two, during which about $50 \%$ ultimately match. The rest take a year off or participate in a final round, "the Scramble," where students apply to unfilled posts.

Those interviewed for this story were quick to say that the problems with the matching service do not lie with CaRMS. Rather, the problems lie with the process itself.

It's also a sensitive issue. Canada's Postgraduate Deans for Medical Education group, the Canadian Association of Internes and Residents (CAIR) and the Professional Association of Residents of Ontario declined to comment on the matching process.

CaRMS Vice-President Irving Gold told CMAJ that students and residents are offered opportunities to provide feedback on the electronic interface, par-

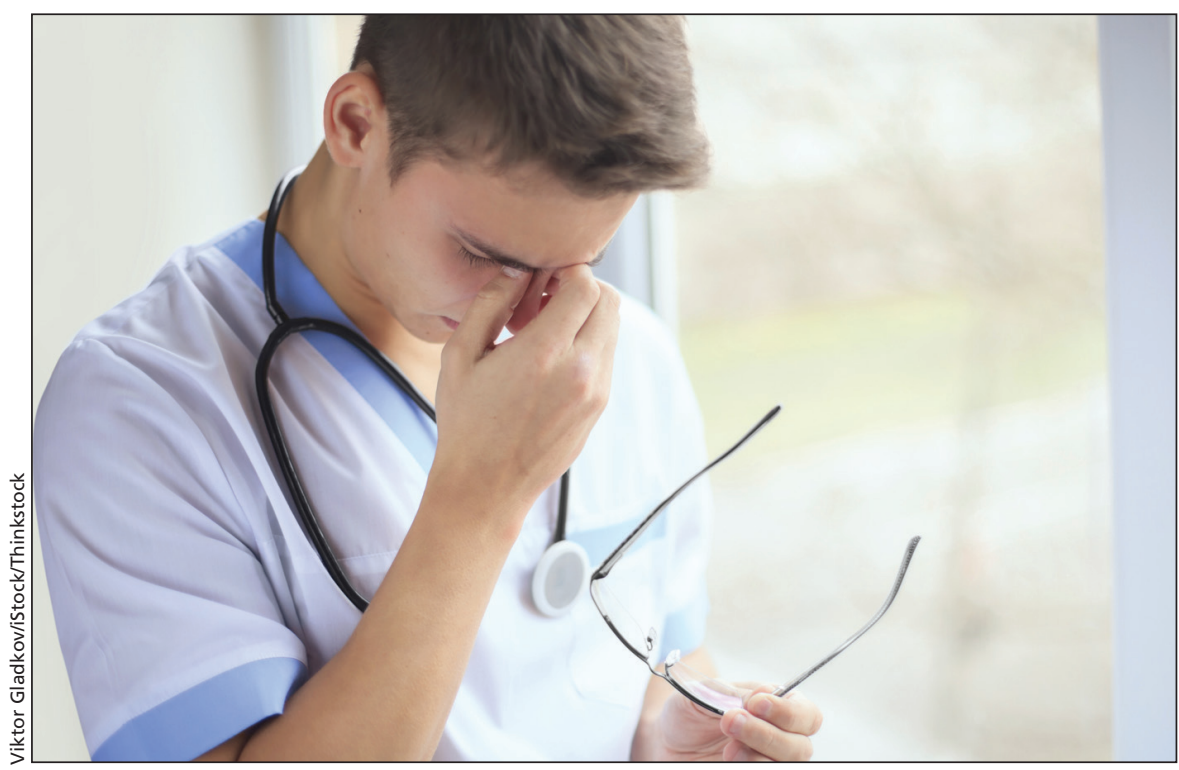

Major concerns in the match process include limited opportunities for electives and a lack of transparency in the selection process.

ticularly after major changes. But Irving focused on discussing the CaRMS website and would not comment on how residents view the matching process as a whole, or on measures of match success.

In contrast, the Canadian Federation of Medical Students (CFMS), was forthright about problems it sees with the match. These problems have reached the point where CFMS plans to study deidentified data on students who go unmatched. "We feel we have a responsibility to help identify any concerning trends and support our members," says Melanie Berchard, vice-president of government affairs.

She says the matching process could be improved by increasing the "provision of objective and easily accessible data describing the characteristics of those students who successfully matched to each residency program."

Transparency is also an issue for a few recently matched residents. "Residency programs do not have a clear rubric on how they select candidates," says Howard Meng, a fourth-year medical student at the University of Toronto who matched this year to anesthesiology. "Although it is mostly based on some combination of factors such as personal statement, reference letters and interview, the weight of any of these components is unknown and applicants do not know how eventual matches are made."

Berchard concurs: "The ability to see the types of electives and degree of research experience of successful applicants would provide students with some direction regarding the qualities that are important to each program." Increased transparency may also combat the "rumours and mythology [that] run rampant throughout medical school classes."

"I am concerned that many of us make significant decisions regarding electives and career direction based on unsubstantiated, anecdotal information," adds Berchard.

In addition to transparency, elective opportunities during medical school were also identified as problematic. "Unfairness within the matching process" begins with elective opportunities, says Dr. Arun Jagdeo, president of the Professional Association of Residents of British Columbia (PAR-BC). "Not all schools are able to offer the same richness or diversity of electives to their medical students. In this respect, students from larger centres may benefit," he says. "And most medical schools will give priority to their own medical students over students from other schools in the electives offering."

Some of the administrative burden of selecting electives may be mitigated by a new online portal being rolled out this 
summer by the Association of Faculties of Medicine of Canada. Its centralized application service and guide to available elective opportunities across Canada will allow students to select the relevant electives and apply for them more easily.

However, the portal won't solve all the problems with the electives process. According to Berchard, "many students feel obligated to pursue electives that will display their strengths to potential residency programs in the match, as opposed to targeting their weaknesses and knowledge gaps."

In addition to the drive for strategic electives, there's the drive to obtain the ideal reference letters and anxiety about interviews and the cost of travel, says Dr. Derek Puddester, special project lead in innovation and evaluation of postgraduate medical education at the University of Ottawa.

The cost of submitting an application to four programs is approximately $\$ 1000$. "Once travel and accommodation are added, the costs can become unwieldy for some," says Jagdeo.

CaRMS itself, seen as user-friendly and efficient by residents, is one of the main strengths in the current system. "CaRMS offers one centralized application system, a one-stop shop where applicants can find out which programs are available and what requirements are in place," says Jagdeo.

The centralized system is an easy way to send multiple applications, as documents uploaded once can be used for any program or school," adds medical student Meng.

\section{United Kingdom and Australia}

Canada's use of a centralized system differs from other countries' matching processes. In the United Kingdom and Australia, for example, trainees apply directly to specialty-specific colleges, which manage their own training programs. Once accepted, trainees rank their choices of participating teaching hospitals, each of which is allocated a specific number of trainees per year.

The UK and Australian training schemes also differ from the Canadian system in that they require a minimum period of working as a general trainee prior to applying for specialty training: two years in the UK and one in Australia.
Dr. Melanie Sahni, a UK graduate who matched to anesthesiology this year, says "the current application process does not require experience in your field of interest, but demonstrating commitment to [a] specialty scores you more points. This was an advantage as I decided on anesthesiology during my two general-training years. In the end, I applied to three different programs to keep my options open."

The UK system is also more transparent. "Person specifications for different specialties and training levels are widely available online, as are marking schemes for applications and interviews. With effort and organization, it's easy to score highly on various stations of the interview," says Sahni. "I received feedback, including where I dropped marks, within two weeks [of] interviews."

In Australia, surgical trainees also know what's required of them at specialty interviews and are allocated jobs based on a points system. "It's a fair, transparent process," says Benjamin Dunne, a cardiothoracic surgical trainee in Perth, Australia. Students also have access to the application marking scheme beforehand and are afforded an opportunity to obtain feedback after interviews, which is useful if they are unsuccessful and wish to reapply.

Like Canada, Australian applicants must pay for travel and accommodation expenses for interviews across the country; there is minimal access to public funds.

"In the digital age of telehealth, Skype, and FaceTime, either we go digital or we go central," says Puddester, who is also a past-president of CAIR. "Colleges should hold interviews at a set time in a set location without interfering with other disciplines."

"The majority of matches are successful and turn out well for both parties. However, there can be a high burden of suffering for all parties," says Puddester. "Improved career planning services from schools and specialty societies and implementation of quality assurance processes would help better Canada's matching process." - Neil Chanchlani, London, UK

CMAJ 2014. DOI:10.1503/cmaj.109-4843 